\title{
PSEUDO-AUTOMORPHISMS AND MOUFANG LOOPS
}

\author{
R. H. BRUCK
}

1. Introduction. A loop $G$ is a system with a binary operation (.) such that: (i) in the equation $x y=z$, any two of $x, y, z$ uniquely determine the third; (ii) $G$ has a unit 1 . The concept of subloop should be clear. A permutation $U$ of $G$ will be called a pseudo-automorphism of $G$ provided there exists at least one element $u$ of $G$ such that

$$
(x y) U \cdot u=x U \cdot(y U \cdot u)
$$

for all $x, y$ of $G$. The element $u$ will be called a companion of $U$. It is readily verified that the pseudo-automorphisms of a loop $G$ form a group under composition. Indeed, $U^{-1}$ has $p$ as a companion, where $p U \cdot u=1$. And, if $V$ has companion $v$, then $V U$ has companion $v U \cdot u$.

A Moufang loop is one which satisfies the identity

$$
x y \cdot z x=x(y z \cdot x) .
$$

An extensive study of Moufang loops is given in [2]. ${ }^{1}$ One defect of that study is that it assumes Moufang's associativity theorem [6], the only published proof of which involves a complicated induction. Using pseudo-automorphisms along with recent methods of Kleinfeld and the author [5], we shall give simple noninductive proofs of three associativity theorems, one of which (Theorem 5.1) generalizes that of Moufang. As shown in [3], still simpler proofs of Moufang's theorem are possible in the commutative case. And, indeed, the following corollary of Theorem 5.3 can be obtained directly from Lemmas 2.1, 2.2: Every associative subset of a commutative Moufang loop $G$ is contained in an associative subloop of $G$.

The present methods represent a considerable improvement over those of [2] (in particular, pseudo-automorphisms have displaced the cumbersome autolopisms) and the paper should serve as an introduction to the theory of Moufang loops. There is little overlapping, except possibly in $\$ 2$, but we have added (Theorem 4.1) a more aesthetic proof of the fact that the nucleus (previously called the associator) of a Moufang loop is a normal subloop.

2. Elementary properties. Henceforth let $G$ be a Moufang loop. From (1.2) with $z=1$,

Received by the editors July 18, 1950 and, in revised form, May 18, 1951.

1 Numbers in brackets refer to the bibliography at the end of the paper. 


$$
x y \cdot x=x \cdot y x .
$$

Defining the inverse $x^{-1}$ of $x$ by $x x^{-1}=1$, we set $y=x^{-1}$ in (2.1) and find $x=x \cdot x^{-1} x, 1=x^{-1} x$. Thus, also, $\left(x^{-1}\right)^{-1}=x$. Setting $z=x^{-1}$ in (1.2), we have $x y=x\left(y x^{-1} \cdot x\right), y x^{-1} \cdot x=y$. Again, from (1.2), (2.1), $z x=x x^{-1} \cdot z x=\left(x \cdot x^{-1} z\right) x, z=x \cdot x^{-1} z$. Therefore

$$
x^{-1} \cdot x y=y=y x \cdot x^{-1} \text {. }
$$

If $z=x y$, then, by (2.2), $z y^{-1}=x, z^{-1} x=y^{-1}, y^{-1} x^{-1}=z^{-1}$; thus

$$
(x y)^{-1}=y^{-1} x^{-1} \text {. }
$$

Setting $y=p z^{-1}$ in (1.2), and using (2.2), (2.1), we get $\left(x \cdot p z^{-1}\right)(z x)$ $=x p \cdot x$, whence, by (2.2), (2.3), $x \cdot p z^{-1}=(x p \cdot x)\left(x^{-1} z^{-1}\right)$. From this, with $z^{-1}=x q, x(p \cdot x q)=(x p \cdot x) q$. Therefore, in view of (2.3), we have the Moufang identities,

$$
x(y \cdot x z)=(x y \cdot x) z, \quad(z x \cdot y) x=z(x \cdot y x) .
$$

As Bol [1] was first to show, each of the identities (2.4) implies (1.2).

For each $a$ of $G$, define permutations $L(a), R(a)$ of $G$ by $x L(a)=a x$, $x R(a)=x a$. In view of (2.2),

$$
L(x)^{-1}=L\left(x^{-1}\right), \quad R(x)^{-1}=R\left(x^{-1}\right) .
$$

In this notation, (1.2) becomes

$$
y L(x) \cdot z R(x)=(y z) R(x) L(x) .
$$

Again, setting $x=p^{-1}, y=q^{-1}, z=q r$ in (1.2), we have $\left(p^{-1} q^{-1}\right)\left(q r \cdot p^{-1}\right)$ $=p^{-1} \cdot r p^{-1}$ and hence $(q p)\left(p^{-1} \cdot r p^{-1}\right)=q r \cdot p^{-1}$. Equivalently,

$$
y R(x) \cdot z R\left(x^{-1}\right) L\left(x^{-1}\right)=(y z) R\left(x^{-1}\right) .
$$

Let $\&$ be the permutation group generated by all the $L(x), R(x)$, and let $\Im$ be the subgroup consisting of those $U$ of $\&$ such that $1 U=1$. An element of $\Im$ is known as an inner mapping of the loop $G$. We are ready to prove a basic lemma.

Lemma 2.1. Every inner mapping of a Moufang loop $G$ is a pseudoautomorphism of $G$.

Proof. In view of (2.5), every element $U$ of $\&$ has the form $U=U_{1} U_{2} \cdots U_{n}$ where $U_{i}=L\left(a_{i}\right)$ or $R\left(a_{i}\right)$ for an element $a_{i}$ of $G$. We see from (2.6), (2.7) that for each $i$ there exist elements $V_{i}, W_{i}$ of (5) such that $x U_{i} \cdot y V_{i}=(x y) W_{i}$ for all $x, y$ of $G$. Thus, if $V$ $=V_{1} V_{2} \cdots V_{n}, W=W_{1} W_{2} \cdots W_{n}$,

$$
x U \cdot y V=(x y) W
$$


for all $x, y$ of $G$. If $U$ is in $\Im, 1 U=1$, so that (2.8) with $x=1$ gives $V=W$. Now set $u=1 V$. Then (2.8) with $y=1$ gives $x U \cdot u=x W$, so that (2.8) becomes (1.1). We shall use this proof as a method of computing a companion of an inner mapping.

A better result holds for commutative Moufang loops:

LEMMA 2.2. Every pseudo-automorphism of a commutative Moufang loop $G$ is an automorphism of $G$.

Proof. If $G$ is commutative, interchange of $x$ and $y$ in (1.1) gives $x U \cdot(y U \cdot u)=y U \cdot(x U \cdot u)$. Setting $x U=p, y U=q u^{-1}$ in this, we get $p q=\left(q u^{-1}\right)(p u)=p u \cdot q u^{-1}$, whence, by (2.4), $p q \cdot u=\left(p u \cdot q u^{-1}\right) u=p \cdot u q$ $=p \cdot q u$ for all $p, q$, of $G$. Therefore (1.1) yields $(x y) U \cdot u=(x U \cdot y U) u$, $(x y) U=x U \cdot y U$.

If $h\left(x_{1}, \cdots, x_{n}\right)$ is a single-valued function from $G$ to $G$, and if $A_{i}$ $(i=1, \cdots, n)$ are subsets of $G, h\left(A_{1}, \cdots, A_{n}\right)$ denotes the set of all elements $h\left(a_{1}, \cdots, a_{n}\right)$ with $a_{i}$ in $A_{i}$. Subsets consisting of one element will usually be denoted by that element. Note the meanings of $A^{-1}, A^{2}, A A$.

3. Invariant elements of pseudo-automorphisms. In (1.1) set $x=y$ $=1$ and get $1 U \cdot u=1 U \cdot(1 U \cdot u), 1=1 U$. Then set $y=x^{-1}$ and get $u=x U \cdot\left(x^{-1} U \cdot u\right)$,

$$
1 U=1, \quad x^{-1} U=(x U)^{-1} .
$$

Next replace $y$ in (1.1) by $y x$ and use (2.1), (1.1), (2.4) to get $(x y x) U \cdot u$ $=x U \cdot((y x) U \cdot u)=x U \cdot(y U \cdot(x U \cdot u))=(x U \cdot y U \cdot x U) u$,

$$
(x y x) U=x U \cdot y U \cdot x U \text {. }
$$

The identity (3.2) states that every pseudo-automorphism of a Moufang loop $G$ is a semi-automorphism of $G$. And it is easily seen that (3.2) implies (3.1). In the following theorem, we could use semi-automorphisms, but pseudo-automorphisms seem more natural.

THEOREM 3.1. Let $\mathfrak{S}$ be any set of pseudo-automorphisms of a Moufang loop $G$. Let $F=F(\mathfrak{S})$ be the set of all $x$ of $G$ left fixed by $\mathfrak{S}$, and let $M=M(\Im)$ be the set of all $m$ of $G$ such that $m F \subset F$. Then: (i) 1 is in $F$; (ii) $F^{-1}=F$ and $x F x=F$ for $x$ in $F$; (iii) $M$ is a subset of $F$ and a subloop of $G$; (iv) $m F=F=F m$ for every $m$ of $M$.

Corollary. If $G$ is also commutative, $M=F$.

Proof. (i), (ii) reflect (3.1), (3.2). By (i), $F$ contains $M \cdot 1=M$. If $m$ is in $M, x$ in $F, x^{-1} m^{-1}=(m x)^{-1}$ is in $F^{-1}=F$, and thus $m^{-1} x$ $=x\left(x^{-1} m^{-1}\right) x$ is in $x F x=F$. Hence $M^{-1}=M$. Moreover, $F=m \cdot m^{-1} F$ 
$\subset m F \subset F$, so that $m F=F$, and, similarly, $F m=F$. If also $m^{\prime}$ is in $M$, $m m^{\prime} \cdot x=\left(m m^{\prime}\right)\left(x m^{-1} \cdot m\right)=m\left(m^{\prime} \cdot x m^{-1}\right) m$ is in $m F m=F$. Hence $M M$ $\subset M$. This is enough to show that $M$ is a subloop of $G$. If $G$ is commutative, Lemma 2.2 shows that $F F \subset F, M=F$, proving the corollary.

4. Some basic lemmas. The commutator $(x, y)$ and associator $(x, y, z)$ of a loop $G$ are defined by

$$
x y=(y x)(x, y), \quad x y \cdot z=(x \cdot y z)(x, y, z) .
$$

Lemma 4.1. In a Moufang loop $G$, the equation $(a, b, c)=1$ implies all of the equations obtained by permuting the elements $a, b, c$ and replacing any of these elements by their inverses.

Proof (cf. [6]). We give a proof which illustrates the use of Theorem 3.1. Assume that

$$
(a, b, c)=1, \quad a b \cdot c=a \cdot b c .
$$

Clearly (4.2) can be written in the form $a U=a$ where $U$ $=R(b) R(c) R(b c)^{-1}, 1 U=1$. By Lemma $2.1, U$ is a pseudo-automorphism. Thus, by Theorem 3.1 (ii), (4.2) implies $\left(a^{-1}, b, c\right)=1$. Similarly, (4.2) implies $\left(a, b^{-1}, c\right)=\left(a, b, c^{-1}\right)=1$. Also (4.2) implies $c^{-1} \cdot b^{-1} a^{-1}=c^{-1} b^{-1} \cdot a^{-1}$ and hence $\left(c^{-1}, b^{-1}, a^{-1}\right)=1=(c, b, a)$. Next, from $\left(a^{-1}, b, c\right)=1$, we get $a^{-1} b \cdot c=a^{-1} \cdot b c, b c \cdot a=a\left(a^{-1} \cdot b c\right) a$ $=a\left(a^{-1} b \cdot c\right) a=b \cdot c a,(b, c, a)=1$. This completes the proof.

Lemмa 4.2. Let $a, b, c, d$ be elements of a Moufang loop $G$, each three of which associate (satisfy $(x, y, z)=1)$. Then the following equations are equivalent: (i) $(a, b, c d)=1$; (ii) $(c, d,(a, b))=1$; (iii) $\left(c, d,(a b)^{2}\right)=1$; (iv) $(c, d, a b)=1$; (v) $(d, a, b c)=1$. Hence (i) is equivalent to each of the equations obtained by permuting the elements $a, b, c, d$ and replacing any of these elements by their inverses.

Proof (cf. [5, Lemma 2.1]). By Lemma 4.1, the equation $(a, b, x)$ $=1$ is equivalent to $\left(b^{-1}, a^{-1}, x\right)=1$. The latter may be written as $x U=x$ where $U=L\left(a^{-1}\right) L\left(b^{-1}\right) L(a b)$. Using the proof of Lemma 2.1, we see that $U$ has companion $u=1 R\left(a^{-1}\right) R\left(b^{-1}\right) R(a b)=(a, b)$. Then $a b=b a \cdot u, a b a=(b a \cdot u) a=b(a u a)$. Therefore $b(a u a) b=a b a \cdot b$ $=(a b \cdot a)\left(a^{-1} \cdot a b\right)=(a b)\left(a a^{-1}\right)(a b)$,

$$
b(a u a) b=(a b)^{2} .
$$

Now (i) is equivalent to $c d \cdot u=(c d) U \cdot u=c U \cdot(d U \cdot u)=c \cdot d u,(c, d, u)$ $=1$, or (ii). Since $(c, d, x)=1$ is equivalent to $x V=x$ for an inner mapping $V,(4.3)$ and Theorem 3.1 (ii) show that (ii) is equivalent to 
(iii). Hence (i) is equivalent to (iii). Similarly (iv) is equivalent to $\left(a, b,(c d)^{2}\right)=1$. However, since $x \cdot 1 \cdot x=x^{2}$, (i) implies $\left(a, b,(c d)^{2}\right)=1$ and (iv). Conversely (iv) implies (i); together they imply $(a \cdot b c) d$ $=(a b \cdot c) d=a b \cdot c d=a(b \cdot c d)=a(b c \cdot d),(a, b c, d)=1$, or, (v). And if (i) implies (v), then, equally, (v) implies (i). This, together with Lemma 4.1, suffices for the proof of Lemma 4.2.

If $A$ is any subset of a Moufang loop $G$, we define the adjoint $A^{\prime}$ of $A$ in $G$ as the set of all $c$ in $G$ such that $(A, c, G)=1$. We define the closure $A^{*}$ of $A$ in $G$ by $A^{*}=\left(A^{\prime}\right)^{\prime}$. In view of Lemma 4.1 , the closure has the usual properties: (i) $A \subset A^{*}$; (ii) $A^{* *}=A^{*}$; (iii) if $A \subset B$, $A^{*} \subset B^{*}$.

Lemma 4.3. The adjoint $A^{\prime}$ and closure $A^{*}$ of a subset $A$ of a Moufang loop $G$ are subloops of $G$, and $A \subset A^{*}$. Moreover, $(A, A, G)=1$ implies $\left(A^{*}, A^{*}, G\right)=1$.

Proof (cf. [8]). Let $B=A^{\prime}$. By Theorem 3.1, $B^{-1} \subset B$. For $a$ in $A$, $b, b^{\prime}$ in $B, x$ in $G$, we have, by three uses of the definition and two uses of (2.4), $\left(\left(a \cdot b^{\prime} b\right) x\right) b=\left(\left(a b^{\prime} \cdot b\right) x\right) b=\left(a b^{\prime}\right)(b x b)=a\left(b^{\prime} \cdot b x b\right)=a\left(\left(b^{\prime} b \cdot x\right) b\right)$ $=\left(a\left(b^{\prime} b \cdot x\right)\right) b$. Therefore $\left(a \cdot b^{\prime} b\right) x=a\left(b^{\prime} b \cdot x\right),(A, B B, G)=1, B B \subset B$. Hence $B$ is a subloop. Since $A^{*}=B^{\prime}, A^{*}$ is also a subloop. If $(A, A, G)$ $=1$, then $A \subset A^{\prime}$. Hence $\left(A, A^{*}, G\right)=1, A^{*} \subset A^{\prime}$. Thus, finally, $\left(A^{*}, A^{*}, G\right)=1$.

The nucleus $N$ of a Moufang loop $G$ is the set of all $n$ of $G$ such that $(n, G, G)=1$. (In $[2,4]$ and elsewhere, $N$ is called the associator.)

Theorem 4.1. If $G$ is a Moufang loop with nucleus $N$, every pseudoautomorphism of $G$ induces an automorphism of $N$. In particular, $N$ is a characteristic normal subloop of $G$.

Proof (cf. [2]). Since $N=G^{\prime}, N$ is a subloop. If $n$ is in $N$, and $U$ is any pseudo-automorphism, let $a=n U, V=U^{-1}$. Then $(a x) V \cdot v=n$ $\cdot(x V \cdot v)=(n \cdot x V) v$ or $(a x) V=n \cdot x V$ for every $x$ of $G$. Hence $(a x \cdot y) V \cdot v$ $=(a x) V \cdot(y V \cdot v)=(n \cdot x V)(y V \cdot v)=n(x V \cdot(y V \cdot v))=n \cdot((x y) V \cdot v)=(n$ $\cdot(x y) V) v=(a \cdot x y) V \cdot v, a x \cdot y=a \cdot x y, a \in N, N U \subset N$. Then $N=(N V) U$ $\subset N U \subset N, N U=N$. And, for $n, n^{\prime}$ in $N,\left(n U \cdot n^{\prime} U\right) V=n \cdot n^{\prime} U V$ $=n n^{\prime}$, or $n U \cdot n^{\prime} U=\left(n n^{\prime}\right) U$. This proves the first sentence. Since $N \Im$ $=N, N$ is normal. (See Lemma 2.1 and the theory of normality in [2].) And since automorphisms are pseudo-automorphisms, $N$ is characteristic.

5. Associativity theorems. In view of (2.1) and Lemma 4.1, we have $(x, x, G)=1$ for every element $x$ of the Moufang loop $G$.

Theorem 5.1. Let $A, B, C$ be subsets of a Moufang loop $G$ such that 
$(A, A, G)=(B, B, G)=(C, C, G)=(A, B, C)=1$. Then the subset $D=A \cup B \cup C$ is contained in an associative subloop $H$ of $G$.

Corollary, Any two elements $a, b$ of a Moufang loop $G$ (or any three elements $a, b, c$ such that $a b \cdot c=a \cdot b c)$ are contained in an associative subloop of $G$.

Proof (cf. $[3,5,6,7,8]$ ). Let $F$ be the set of all elements $x$ in $G$ such that $(D, D, x)=(A B, C, x)=1$, and let $M$ be the set of all $m$ in $G$ such that $m F \subset F$. By Theorem 3.1, $M$ is a subloop of $G$ such that $(D, D, M)=1$. In view of Lemmas 4.1, 4.2, $A, B$, and $C$ play symmetrical rôles in the definition of $F$. We now use Lemma 4.2 four times along with Lemma 4.1. Since $(A, A, D)=(A, A, F)=(A, A, D F)$ $=1$, then also $(A A, D, F)=(A D, A, F)=(D A, A, F)=1$. From this, and by symmetry, $(D D, D, F)=1$, and hence $(D D, A, F)=1$. Since $(D, D, D)=(D, D, F)=(D D, D, F)=1$, then $(D, D, D F)=1$. In particular, $(D, D, A F)=(D, A, A F)=1$. Since $(A, A, D D)=(A, A, F)$ $=(D D, A, F)=(A, A, D D, F)=1$, also $(D D, A, A F)=1$. And, since $(D, D, A)=(D, D, A F)=(D, A, A F)=(D D, A, A F)=1$, then $(A D, D, A F)=1$. In particular, $(A B, C, A F)=1$. Thus $(D, D, A F)$ $=(A B, C, A F)=1, A \subset M$. By symmetry, $D \subset M$, and we may take $H$ to be the closure of $D$ in $M$. For the corollary, set $A=a, B=C=b$ or $A=a, B=b, C=c$ according to the case.

A subset $A$ of the Moufang loop $G$ is called associative if $(A, A, A)$ $=1$. An associative subset (subloop) $A$ is called a maximal associative subset (subloop) provided $A$ is contained in no associative subset (subloop) of $G$ distinct from $A$. On the basis of Zorn's Lemma, it is clear that every associative subset (subloop) is contained in at least one maximal associative subset (subloop).

TheOREM 5.2. Let $A$ be an associative subloop of a Moufang loop $G$, and let $B$ be a subset of $G$ such that $(A, A, B)=(B, B, G)=1$. Then the subset $D=A \cup B$ is contained in an associative subloop $H$ of $G$.

Corollary. Every maximal associative subloop of a Moufang loop $G$ is a maximal associative subset of $G$.

Proof (cf. [5]). Let $F$ be the set of all $x$ in $G$ such that $(D, D, x)=1$, and let $M$ be the set of all $m$ in $G$ such that $m F \subset F$. By Theorem 3.1, $M$ is a subloop of $G$ such that $(D, D, M)=1$. Since $A A=A,(A, A, D)$ $=(A, A, F)=(A, D, F)=(A A, D, F)=1$, and hence $(A, D, A F)$ $=(A, A, D F)=1$. Since $(B, B, A F)=1$, we have $(D, D, A F)=1$, $A \subset M$. Since $(B, B, D)=(B, B, F)=(B, D, F)=(B, B, D F)=1$, then $(B, D, B F)=1$. Since $(A, A, D F)=1$, then $(A, A, B F)=1$. Therefore 
$(D, D, B F)=1, B \subset M$. Hence $D \subset M$, and we may take $H$ to be the closure of $D$ in $M$. If $A$ is a maximal associative subloop, the relations $A \subset D \subset H$ imply $D=A, B \subset A$; the case $B=b$ shows that $A$ is a maximal associative subset.

Theorem 5.3. Let $G$ be a Moufang loop such that $(G, G,(G, G))=1$. Then every maximal associative subset $A$ of $G$ is a maximal associative subloop of $G$.

REMARK. If $G$ has nucleus $N$, the condition $(G, G,(G, G))=1$ means that $(G, G) \subset N$, or that the quotient loop $G / N$ is commutative. As M. F. Smiley has pointed out (private communication), there exist Moufang loops $G$ for which the conclusion of Theorem 5.3 is false.

Proof (cf. $[3,5]$ ). By Lemma 4.2 , for $a, b, c, d$ in $A$, the valid equation $(c, d,(a, b))=1$ implies $(a, b, c d)=1$. Hence $(A, A, A A)=1$. Thus, for $x$ in $A A, A \cup x$ is an associative subset, $x \in A, A A \subset A$. Similarly, by Theorem 4.1, $A^{-1}=A$. This completes the proof.

\section{BIBLIOGRAPHY}

1. G. Bol, Geweben und Gruppen, Math. Ann. vol. 114 (1937) pp. 414-431.

2. R. H. Bruck, Contributions to the theory of loops, Trans. Amer. Math. Soc. vol. 60 (1946) pp. 245-354.

3. - On a theorem of $R$. Moufang, Proceedings of the American Mathematical Society vol. 2 (1951) pp. 144-145.

4. - An extension theory for a certain class of loops, Bull. Amer. Math. Soc. vol. 57 (1951) pp. 11-26.

5. R. H. Bruck and Erwin Kleinfeld, The structure of alternative division rings, Proceedings of the American Mathematical Society vol. 2 (1951) pp. 878-890.

6. Ruth Moufang, Zur Struktur von Alternativkörpern, Math. Ann. vol. 110 (1935) pp. $416-430$.

7. M. F. Smiley, The radical of an alternative ring, Ann. of Math. vol. 49 (1948) pp. 702-709.

8. Max Zorn, Theorie der alternativen Ringe, Abh. Math. Sem. Hamburgischen Univ. vol. 8 (1931) pp. 123-147.

UNIVERSITY OF WISCONSIN 